University of Warwick institutional repository: http://go.warwick.ac.uk/wrap This paper is made available online in accordance with publisher policies. Please scroll down to view the document itself. Please refer to the repository record for this item and our policy information available from the repository home page for further information.

To see the final version of this paper please visit the publisher's website. Access to the published version may require a subscription.

Author(s): P. D. Quinn, N. R. Wilson, S. A. Hatfield, C. F. McConville, G. R. Bell, T. C. Q. Noakes, P. Bailey, S. Al-Harthi, and F. Gard Article Title: Composition profiles of InAs-GaAs quantum dots determined by medium-energy ion scattering Year of publication: 2005

Link to published version: http://dx.doi.org/10.1063/1.2099533

Publisher statement: None 


\title{
Composition profiles of InAs-GaAs quantum dots determined by medium-energy ion scattering
}

\author{
P. D. Quinn, N. R. Wilson, S. A. Hatfield, C. F. McConville, and G. R. Bell ${ }^{\text {a) }}$ \\ Department of Physics, University of Warwick, Coventry CV4 7AL, United Kingdom \\ T. C. Q. Noakes and P. Bailey \\ CCLRC Daresbury Laboratory, Daresbury, Warrington Cheshire WA4 4AD, United Kingdom \\ S. Al-Harthi and F. Gard \\ Department of Physics, Sultan Qaboos University, PO Box 36, Code 123, Sultanate of Oman
}

(Received 1 June 2005; accepted 8 September 2005; published online 6 October 2005)

\begin{abstract}
The composition profile along the [001] growth direction of low-growth-rate InAs-GaAs quantum dots (QDs) has been determined using medium-energy ion scattering (MEIS). A linear profile of In concentration from $100 \%$ In at the top of the QDs to $20 \%$ at their base provides the best fit to MEIS energy spectra. (C) 2005 American Institute of Physics. [DOI: 10.1063/1.2099533]
\end{abstract}

InAs quantum dots (QDs) grown by self-assembly on $\mathrm{GaAs}(001)$ substrates continue to attract great interest due to their applicability in optoelectronic devices. The optical properties of a QD depend on the detailed shape of its potential well, which can be determined only if the distribution of In in the In/Ga sublattice is known. For the InAs-GaAs system, it is well established that (a) an alloyed InGaAs wetting layer (WL) is first formed during InAs deposition; ${ }^{1}$ (b) the final state of the QDs may be an alloy even for deposition of pure InAs; ${ }^{2}$ and (c) further changes to QD shape and composition take place during the capping of a QD array with GaAs or InGaAs. ${ }^{3-5}$ These processes are controlled by a complicated combination of surface segregation ${ }^{6}$ and locally strain-dependent surface migration and attachment. ${ }^{5}$ Several experimental techniques have been used to probe the In distribution in QDs, including grazing incidence x-ray diffraction (XRD), ${ }^{7}$ cross-sectional scanning tunneling microscopy (XSTM), ${ }^{5,8}$ transmission electron microscopy (TEM) and scanning TEM energy dispersive X-ray analysis. ${ }^{9}$ Both XRD and XSTM suggest that the In composition varies with height through the QDs, with pure InAs at the top of the QDs and progressively more $\mathrm{Ga}$ in the alloy towards the base of the QDs. ${ }^{7,8}$ This alloying effect depends on growth conditions, particularly temperature ${ }^{2}$ and growth rate. ${ }^{10}$

In this letter we describe the use of medium-energy ion scattering (MEIS) to produce composition profiles of InAs QDs. The MEIS technique is able to probe layered materials with near atomic layer resolution. Here we demonstrate its ability to probe an array of three-dimensional (3D) island structures and, when combined with data from atomic force microscopy (AFM), produce compositional profiles along the growth direction.

InAs QDs were grown on $\mathrm{GaAs}(001)$ by molecular beam epitaxy at a substrate temperature of $480{ }^{\circ} \mathrm{C}$. The total amount of InAs deposited was 2.2 monolayers $(1 \mathrm{ML}=$ $0.3 \mathrm{~nm}$ ) and the growth rate was $0.012 \mathrm{ML} \mathrm{s}^{-1} .{ }^{10}$ Here, we discuss free-standing QDs uncapped by GaAs. Samples were transferred through air between the MBE chamber and the MEIS system, with an arsenic capping-decapping procedure a) Electronic mail: gavin.bell@ @hysics.org; URL: http://www.warwick.ac.uk/
go/grbell employed to minimize oxidation. ${ }^{11}$ The arsenic decapping anneal was approximately $400{ }^{\circ} \mathrm{C}$ for $15 \mathrm{~min}$, a temperature well below that used to modify the optical properties of InAs-GaAs QDs. ${ }^{12}$ Residual submonolayer contamination by oxygen and carbon was normally detected by Auger electron spectroscopy, but neither this nor the anneal itself materially affect the In profile results presented here. Ion scattering experiments were performed using the UK MEIS Facility at Daresbury Laboratories. ${ }^{13}$ Helium ions at $100 \mathrm{keV}$ energy were incident a pseudorandom direction near $\langle 110\rangle$. The toroidal electrostatic detector monitors an angular range of $27^{\circ}$ and has an energy resolution $\sim 400 \mathrm{eV}$. In the present experiments the scattering angle was $\sim 90^{\circ}$. Integration of the signal over an appropriate angular range allowed energy spectra to be obtained. For uncapped QDs, the entire In signal at 90-94 keV was separate from the GaAs surface peaks and background, allowing the peak shape to be analyzed independently. Ion beam damage was minimized by regular movement of the sample under the beam. The ion dose at each location $(5 \mu \mathrm{C})$ was below the damage threshold for GaAs measured in separate dechanneling experiments.

An AFM topograph of a typical QD array is shown in Fig. 1. The number density of QDs is $5 \times 10^{10} \mathrm{~cm}^{-2}$ while the mean height and diameter are 2.8 and $27 \mathrm{~nm}$, respectively. Large 3D islands (L3DI) of mean height $8 \mathrm{~nm}$ are present at much lower number density, typically 5 $\times 10^{8} \mathrm{~cm}^{-2}{ }^{10}$

The pseudorandom MEIS energy spectrum in the In region is shown in Fig. 2 with several simulated spectra superimposed on the data. Slightly different models were employed in Figs. 2(a) and 2(b), as explained subsequently. The asymmetric shapes of the peaks in both the experimental and simulated data are caused by the distribution of In through the QDs. Because of the discrete 3D nature of the QDs, a range of different path lengths, and hence stopping and straggling energy losses, is possible for backscattering from In nuclei at a particular depth. This energy loss process was simulated by placing a single model QD, whose size is determined by AFM, in the center of a section of WL whose area is determined by AFM via the QD number density. Our simulations employ an idealized QD shape but are not sensitive to the precise geometrical figure used (e.g., spherical cap versus truncated pyramid). The whole structure is then 


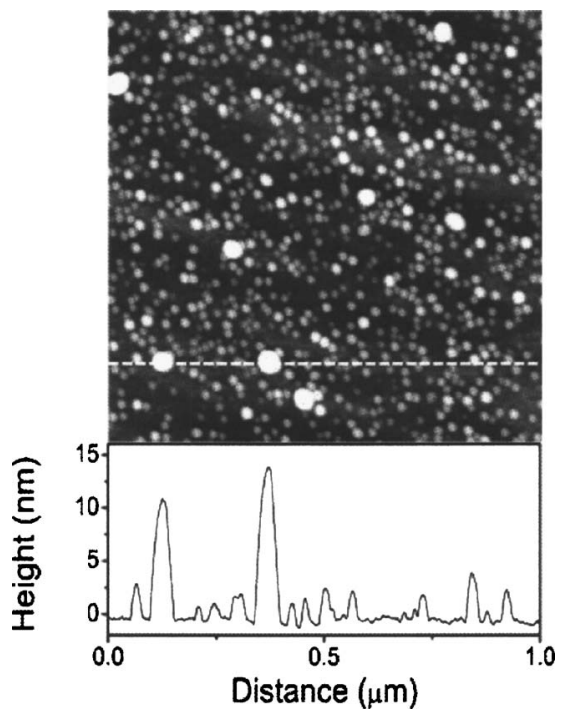

FIG. 1. A $1 \mu \mathrm{m} \times 1 \mu \mathrm{m}$ AFM topograph of uncapped QDs produced by depositing 2.2 ML InAs on GaAs(001). A height cross section shows two L3DIs and several QDs.

divided into a grid of 400 possible ion entry points and the energy spectrum calculated for each of the resulting ion trajectories. The simulated spectrum is a sum over all these possible paths. A similar method has been used for calculating MEIS energy spectra of metal alloy nanoparticles. ${ }^{14}$

We employ the SRIM2000 code and analytic formulae ${ }^{15}$ to obtain stopping powers. The total ion yield $Y$ from element $i$ as a function of energy $E$ is given by ${ }^{16,17}$

$$
Y=x_{i} \sigma_{i} f\left(E-E_{\text {out }} ; \Gamma\right) N(E) \Omega .
$$

In Eq. (1), the Gaussian $f$ is centered on the energy of the ion $E_{\text {out }}$ as it leaves the surface; $E_{\text {out }}$ is given by the appropriate stopping powers and elastic energy loss. The gaussian width $\Gamma$ is the sum of inward and outward straggling terms ${ }^{18}$ and the instrumental resolution. The resolution and detector normalization $\Omega$ are determined by experiments
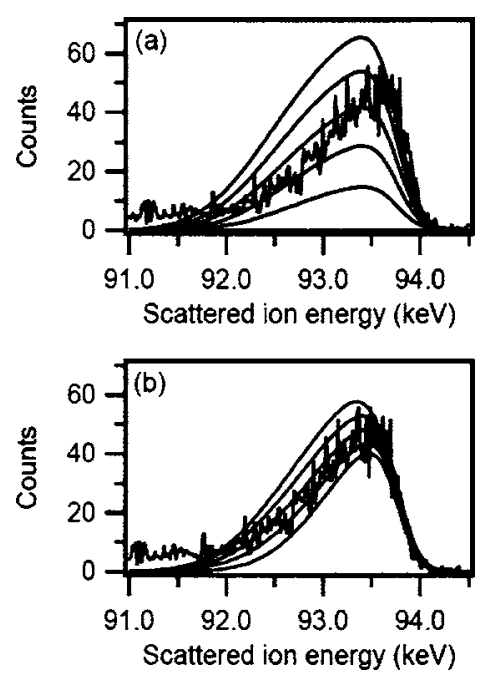

FIG. 2. The MEIS energy spectrum in the In region for uncapped QDs together with illustrative simulations based on fixed QD number density and height, with no L3DI. (a) In compositions constant throughout the QDs are compared; namely, 20\%, 40\%, 60\%, 80\%, and 100\% from bottom to top. (b) Several linear composition profiles are shown, with $100 \%$ In at the top of the QD falling to a value at the base of $0 \%$ (bottom curve), $20 \%, 40 \%, 60 \%$,

or $80 \%$ (top curve).
Downloaded 06 Jul 2009 to 137.205 .202 .8 . Redistribution subject to AlP license or copyright; see http://apl.aip.org/apl/copyright.jsp

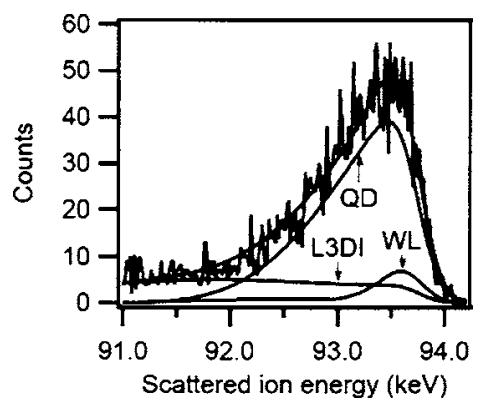

FIG. 3. The MEIS energy spectra from experiments and optimized simulations. The QD, L3DI, and WL contributions to the simulated spectrum are also shown.

on clean metal surfaces. ${ }^{19}$ The atomic density and cross section are given by $x_{i}$ and $\sigma_{i}$, respectively, while the neutralization probability $N(E)$ is determined from tabulated data. ${ }^{16}$

The simulated curves in Fig. 2 do not include any L3DIs and employ fixed parameters to illustrate the sensitivity of the method. In Fig. 2(a), the model QD has a uniform In composition that does not vary with height, while in Fig. 2(b), the In fraction varies linearly from $100 \%$ at the top of the QD to different values at the base. The plots clearly show the sensitivity of the technique to the composition profile. Similar sensitivity to the number density and QD sizes are observed, but these parameters are easily determined by AFM imaging. The simulations allow several parameters to be optimized simultaneously, typically converging in around $10 \mathrm{~h}$ on a standard desktop computer.

Figure 3 shows the best fit to the MEIS energy spectrum including the QD, L3DI, and WL components. The WL composition and the compositions at the top and bottom of the QDs are all free parameters. The WL thickness and L3DI composition are fixed at $2 \mathrm{ML}$ and pure InAs, respectively. A linear variation with height in the QDs is assumed as the simplest nonconstant composition. ${ }^{7,8}$ The contribution of the WL is important, particularly at the leading edge of the energy spectrum. The WL is expected to be an InGaAs alloy of thickness 1-2 ML, ${ }^{1}$ and indeed the optimized WL comprises the WL would result in too steep a leading edge in the energy spectrum. By contrast, the L3DIs contribute a very broad background extending to low ion energies due to the long path lengths available through them. This background is not strongly affected by the size distribution of the L3DIs; the density is more important, and is fixed by AFM. Having accounted for the L3DI and WL contributions, the optimized QD model corresponds to a linear InGaAs profile, from $100 \%$ In at the top to $20 \%$ In at the base. As shown in Fig. 3, an excellent fit can be obtained using the linear profile. Satisfactory fits could not be obtained using any uniform composition: it is clear from Fig. 2 that the shape of the spectrum in the $92-93 \mathrm{keV}$ region is incorrect for uniform In profiles.

It was inferred from total volume measurements ${ }^{10}$ that pure InAs QDs could be obtained for substrate temperatures around $500{ }^{\circ} \mathrm{C}$ by using growth rates $\leqslant 0.02 \mathrm{ML} \mathrm{s}^{-1}$. However, the present study demonstrates clearly that $\mathrm{Ga}$ is still found in significant quantities in the QDs for these growth conditions. Compositional variation is to be expected through nominally pure InAs QDs even when they are grown at low deposition rates. The precise composition profile of QDs should depend on growth conditions because of the
AlP license or copyright; see http://apl.aip.org/apl/copyright.jsp a 2 ML thick $\operatorname{In}_{0.05} \mathrm{Ga}_{0.95}$ As alloy. A higher In composition in 
complex interplay of kinetic factors involved (segregation, migration, attachment). Indeed, in previous studies based on $\mathrm{XSTM}^{8}{ }^{8}$ where the growth rate was $0.01 \mathrm{ML} \mathrm{s}^{-1}$ and the substrate temperature was $512{ }^{\circ} \mathrm{C}$, a linear In profile from $60 \%$ to $100 \%$ was inferred. This result depends on finite element calculations to fit the measured outward relaxation of the QDs and requires that the QDs be fully capped with GaAs. Similarly, the work of Kegel et al. ${ }^{7}$ produced a roughly linear profile from $5 \%$ to $100 \%$ for QDs grown by periodic In supply at an average rate of $0.035 \mathrm{ML} \mathrm{s}^{-1}$ and temperature $530^{\circ} \mathrm{C}$. These XRD studies depend on the QDs being uncapped. By contrast, MEIS provides a direct method for deriving In profiles in InGaAs structures including both capped and uncapped QDs.

One of the authors (G.R.B.) is grateful to the Royal Society for financial support. The authors thank T. S. Jones for access to the MBE growth facility at Imperial College London. The EPSRC is gratefully acknowledged for access to the MEIS facility.

${ }^{1}$ J. Belk, C. McConville, J. Sudijono, T. Jones, and B. Joyce, Surf. Sci. 387, 213 (1997).

${ }^{2}$ P. Joyce, T. Krzyzewski, G. Bell, B. Joyce, and T. Jones, Phys. Rev. B 58, R15981 (1998)

${ }^{3}$ P. Joyce, T. Krzyzewski, G. Bell, and T. Jones, Appl. Phys. Lett. 79, 3615
(2001).

${ }^{4}$ P. Joyce, T. Krzyzewski, P. Steans, G. Bell, J. Neave, and T. Jones, Surf. Sci. 492, 345 (2001).

${ }^{5}$ D. Bruls, P. Koenraad, H. Salemink, J. Wolter, M. Hopkinson, and M. Skolnick, Appl. Phys. Lett. 82, 3758 (2003).

${ }^{6}$ P. Howe, E. L. Ru, R. Murray, and T. Jones, J. Cryst. Growth 278, 57 (2005).

${ }^{7}$ I. Kegel, T. Metzger, A. Lorke, J. Peisl, J. Stangl, G. Bauer, J. Garcia, and P. Petrof, Phys. Rev. Lett. 85, 1694 (2000).

${ }^{8}$ D. Bruls, J. Vugs, P. Koenraad, H. Salemik, J. Wolter, M. Hopkinson, M. Skolnick, F. Long, and S. Gill, Appl. Phys. Lett. 81, 1708 (2002).

${ }^{9}$ P. Siverns, S. Malik, G. McPherson, D. Childs, C. Roberts, R. Murray, B. Joyce, and H. Davock, Phys. Rev. B 58, R10127 (1998).

${ }^{10}$ P. Joyce, T. Krzyzewski, G. Bell, T. Jones, E. L. Ru, and R. Murray, Phys. Rev. B 64, 235317 (2001).

${ }^{11}$ G. Bell, C. McConville, and T. Jones, Phys. Rev. B 54, 2654 (1996).

${ }^{12}$ S. Malik, E. L. Ru, D. Childs, and R. Murray, Phys. Rev. B 63, 155313 (2001).

${ }^{13}$ P. Bailey, T. Noakes, and D. Woodruff, Surf. Sci. 426, 358 (1999), http:// www.dl.ac.uk/MEIS

${ }^{14}$ I. Konimi, S. Hyodo, and T. Motohiro, J. Catal. 192, 11 (2000).

${ }^{15}$ J. Ziegler and J. Biersack, http://www.srim.org

${ }^{16}$ Y. Kido and T. Koshikawa, J. Appl. Phys. 67, 187 (1990).

${ }^{17}$ E. Gusev, H. Lu, T. Gustafsson, and E. Garfunkel, Phys. Rev. B 52, 1759 (1995).

${ }^{18}$ Q. Yang and D. O'Connor, Nucl. Instrum. Methods Phys. Res. B 61, 149 (1991).

${ }^{19}$ D. Brown, P. Quinn, D. Woodruff, P. Bailey, and T. Noakes, Phys. Rev. B 61, $7706(2000)$ 